\title{
Farmacoterapia de la deshabituación alcohólica. Nuevos fármacos, nuevos conceptos
}

Balcells, M. ${ }^{1}$, Gual, A. ${ }^{2}$

(1) Unidad de Alcohología de la Generalitat de Cataluña. ICPP. Hospital Clínico de Barcelona

(2) Unidad de Alcohología de la Generalitat de Cataluña. ICPP. Hospital Clínico de Barcelona. Presidente de Socidrogalcohol

\section{RESUMEN}

Este capítulo revisa la evidencia existente sobre la efectividad de la farmacoterapia en la deshabituación alcohólica. Los fármacos ensayados actúan sobre diversos neurotransmisores cerebrales: dopamina, serotonina, GABA, ácido glutámico, sistema opioide, etc. Disulfiram, acamprosato y naltrexona son los fármacos más comúnmente usados en la deshabituación. El disulfiram parece ser efectivo especialmente cuando se administra supervisadamente. En el caso de la naltrexona y acamprosato, los mecanismos de acción farmacológicos no han podido establecerse plenamente, aunque existe evidencia de que el acamprosato actúa modulando el sistema NMDA-glutamato, mientras que la naltrexona actúa en el sistema de recompensa a través de los opioides endógenos. Asimismo, no se ha podido establecer cual es el perfil clínico de los pacientes que mejor responden a dichos fármacos.

Fármacos que actúan sobre el sistema dopaminérgico (tiapride, bromocriptina, flupentixol), sobre el sistema serotoninérgico (buspirona, ISRS, ondansetron), así como el ácido $\gamma$-hidroxibutírico (GHB), han sido utilizados en ensayos clínicos, sin que la evidencia sea suficiente para aprobar su utilización en la dependencia alcohólica. Finalmente, la investigación realizada con combinaciones de los diversos fármacos citados, no aporta de momento resultados relevantes.

Palabras clave: Alcohol, dependencia, deshabituación, síndrome de abstinencia, craving, disulfiram, acido $\gamma$-hidróxibutírico, naltrexona, acamprosato, nalmefene, bromocriptina, tiapride, flupentixol, ISRS, Buspirona, ondansetron.

\begin{abstract}
This chapter provides an overview of the data supporting the role for pharmacotherapy in the treatment of alcohol dependence. Drugs acting on several brain neurotransmitters systems including dopamine, serotonin, GABA, glutamic acid and opioid systems are under investigation. Disulfiram, naltrexone and acamprosate are the drugs most commonly used during the rehabilitation period. Disulfiram seems to be effective mostly when given under supervision. In the case of acamprosate and naltrexone, the underlying mechanisms of action have not yet been fully established, even though there's evidence that acamprosate acts on the NMDAglutamate system, while naltrexone seems to act on the reward system through the endogenous opioids. Also, the clinical profile of patients who might benefit the most from each of those drugs is still unknown.

Drugs acting on dopaminergic mechanisms (tiapride, bromocriptin, flupenthixol), serotonergic mechanisms (buspirone, SSIR, ondansetron, buspiron) and $\gamma$-hidroxibutyric acid, have been studied in clinical trials but have not shown sufficient evidence to be approved to treat alcohol dependence. Further research with drug combinations has also been conducted, but results are not conclusive.
\end{abstract}

Key words: Alcohol, dependence, craving, disulfiram, $\gamma$-hidroxibutiric acid, naltrexone, acamprosate, nalmefene, bromocriptin, tiapride, flupenthixol, SSRI, buspirone, ondansetron. 


\section{INTRODUCCIÓN}

E I conocimiento y el número de investigaciones científicas y clínicas sobre el alcoholismo se ha incrementado espectacularmente en la última década, mientras que el tratamiento del alcoholismo está tan solo ahora, empezando a cambiar. En la actualidad, se conocen mejor los trastornos neuroquímicos subyacentes a los procesos de adicción y a las anomalías psiquiátricas (depresión, ansiedad, deterioro neurocognitivo, etc.) que frecuentemente se asocian al alcoholismo. Todo ello, ha favorecido la aparición de nuevos fármacos eficaces en la fase de deshabituación alcohólica que se complementan con los clásicos fármacos antidipsotropos como el disulfiram o la cianamida cálcica y ha empezado ha mejorar el pronóstico a largo plazo de esta compleja enfermedad.

Así mismo, es interesante remarcar cómo, de forma muy significativa, el concepto de enfermedad alcohólica y las distintas hipótesis sobre la etiopatogenia de la dependencia alcohólica han ido justificando y condicionando de forma directa o indirecta el tratamiento del alcoholismo.

Cuando se considera la dependencia alcohólica únicamente como una consecuencia directa de las capacidades euforizantes y altamente reforzantes del alcohol (teorías del refuerzo positivo), el tratamiento se centra en evitar este refuerzo placentero, utilizando básicamente fármacos antidipsotrópicos como el disulfiram o la cianamida cálcica. Cuando la hipótesis que prevalece apunta que el consumo de alcohol se mantiene en el tiempo debido a la aparición de tolerancia y síndrome de abstinencia (teorías del refuerzo negativo), el tratamiento se dirige a paliar o evitar el síndrome de abstinencia y se centra en la desintoxicación del paciente alcohólico. Así mismo, de forma más reciente aparecen las nuevas teorías de la sensibilización neuronal ${ }^{1}$ que postulan que una vez instaurada la adicción esta se vehiculiza a través de una vía neuronal especifica responsable del deseo patológico e irrefrenable de consumo o craving. Las estrategias terapéuticas se basarán entonces consecuentemente en modificar o revertir estas lesiones o disfunciones neurológicas utilizando los nuevos fármacos anticraving.

\section{FÁRMACOS ANTIDIPSOTRÓPICOS O AVERSIVOS}

Los fármacos antidipsotrópicos o aversivos como el disulfiram y la cianamida cálcica han sido los fármacos mas usados en el tratamiento del alcoholismo y siguen teniendo una amplia vigencia en la actualidad. Estos fármacos interfieren el metabolismo del alcohol, inhiben la acción de la aldehidodeshidrogenasa y provocan un incremento de los niveles plasmáticos de acetaldehido responsable de la sintomatología de taquicardia, hipotensión, rubefacción, cefalea, etc.

Sorprendentemente el número de estudios que prueban su eficacia es escaso y como comentan Hughes y Cook ${ }^{2}$ en su revisión de 34 estudios realizados entre 1967 y 1995, en muchos casos presentan problemas metodológicos o fallos estadísticos. En general, parece demostrado que la eficacia de los fármacos aversivos mejora si se consigue aumentar la adhesión al tratamiento con supervisión por parte de un familiar o persona responsable $^{3} \mathrm{o}$ asociando el disulfiram a otros programas de tratamiento, tanto psicosocial ${ }^{2,3}$ como farmacológico ${ }^{4,5}$.

El mecanismo terapéutico de acción de los fármacos aversivos parece residir en el miedo a la reacción disulfiram-alcohol mas que en la propia acción farmacológica per se. Esto explicaría porque en estudios como el de Wilson y colaboradores ${ }^{6,7}$ la abstinencia fue igual en el grupo placebo que en el de disulfiram pero mayor que en el grupo control sin fármaco. Uno de los problemas metodológicos relevantes con estos fármacos lo constituye la definición de la abstinencia como variable principal, cuando las diferencias frente a placebo sólo son esperables precisamente en aquellos pacientes que rompan la abstinencia y, por tanto, experimenten la reacción 
aversiva frente al alcohol ${ }^{8}$. Así mismo la baja eficacia a largo plazo de estos fármacos se explica también por su falta de acción farmacológica per se, pues, actúan sólo como castigo, sin incidir o modificar la dependencia, perdiendo su eficacia inmediatamente después de su retirada, si no van acompañados del correspondiente tratamiento psicosocial.

\section{FÁRMACOS ANTI-CRAVING}

Estos fármacos a los que clasificaremos según el sistema neurotransmisor sobre el que inciden, siguiendo la clasificación utilizada por Soyka ${ }^{9}$, actúan disminuyendo o anulando el deseo compulsivo o imperioso de alcohol o craving. A pesar de ello, sigue siendo necesario recordar que el concepto de craving está poco sistematizado, no hay aún un consenso claro en cuanto a su verdadera implicación en el abuso de alcohol ni sabemos cuales son todos los mecanismos que participan en el deseo irrefrenable de beber. El craving es, a pesar de su inexactitud y dificultad de objetivar y medir, el fenómeno con el que se pretende explicar la posible eficacia de todos estos nuevos fármacos.

\subsection{Fármacos Gabaérgicos y de acción sobre el glutamato -NMDA}

Diversos estudios farmacológicos han demostrado que el alcohol a corto plazo aumenta las acciones GABA (neurotransmisor inhibitorio) sobre sus receptores y disminuye las de los aminoácidos excitatorios, tales como el glutamato, sobre los receptores NMDA $^{10}$. Simplificando, podemos decir que el alcohol reduce la frecuencia de los impulsos eléctricos y deprime la actividad del SNC.

Cuando la exposición al alcohol es crónica, el organismo se adapta disminuyendo la actividad del sistema Gabaérgico inhibidor y aumentando la actividad glutamatérgica excitatoria. El resultado final sería un aumento de la excitabilidad neuronal que contrarrestaría los efectos depresores del alcohol. Al suprimir el consumo de alcohol, el efecto depresor desaparece pero, las neuronas permanecen hiperexcitables. Esta hiperexcitabilidad que requeriría tiempo para readaptarse, sería la responsable de la aparición de síntomas como la ansiedad, el insomnio y el craving.

\section{- Ácido $\gamma$-hidroxibutirico (GHB)}

El GHB es un metabolito del Gaba con actividad neurotransmisora y neuromoduladora. Se han publicado varios estudios clínicos recientes con GHB en el tratamiento del alcoholismo. Además de los trabajos en forma de estudio abierto ${ }^{11}$ existe un estudio doble ciego contra placebo ${ }^{12}$ que parece demostrar un efecto en la disminución del craving y aumento en las tasas de abstinencia con este fármaco. El tamaño de la muestra y el corto tiempo de seguimiento impiden aún sacar conclusiones sobre su eficacia terapéutica. Así mismo, deben tenerse en cuenta la posible capacidad adictiva, los importantes efectos secundarios asociados a este fármaco y la posibilidad de sobredosis s,14,15. $^{13}$.

En un último estudio del año 2000, Moncini, estudia 321 pacientes y encuentra una mejoría sobre placebo en deshabituación pero, así mismo, detecta un no despreciable porcentaje de pacientes que presentaron abuso del fármaco ${ }^{16}$.

\section{- Acamprosato}

El acamprosato es un derivado simple del aminoácido esencial taurina: el ácido acetilhomotaurínico. Presenta actividad como agonista GABA y como inhibidor a nivel de los receptores glutamatérgicos tipo NMDA aunque su mecanismo exacto de acción estaría aun por determinar ${ }^{17,10}$.

Se han realizado con este fármaco un importante número de estudios clínicos desde su introducción en $1985^{18}$. La mayoría de los estudios son doble ciego y demuestran, con un aceptable rigor metodológico ${ }^{19,20}$ una eficacia superior del acamprosato respecto al placebo. En el estudio de Whitworth ${ }^{21}$ se detecta asimismo la persistencia del efecto al cesar 
la administración del fármaco. Aubin ${ }^{22}$ contabiliza mas de dos millones de pacientes tratados en Francia con acamprosato, tres mil de ellos controlados en estudios clínicos y concluye que se ha podido demostrar con claridad la eficacia clínica del acamprosato en: a) la prevención de recaídas alcohólicas a medio y largo plazo, b) un incremento en la adhesión al tratamiento de los pacientes tratados con acamprosato y c) una posible reducción del craving para alcohol.

Se han realizado también estudios multicéntricos en nuestro medio, con resultados igualmente positivos para el acamprosato. El estudio ADISA demostró con una muestra de 288 pacientes y un seguimiento de 6 meses que 1998 mg/día de acamprosato fue más eficaz que placebo para mantener la abstinencia en pacientes alcohol dependientes. El análisis de supervivencia basado en la abstinencia durante todo el estudio obtuvo un porcentaje de éxito del 35\% en pacientes tratados con acamprosato comparado con un $26 \%$ en placebo ${ }^{23}$. Así mismo, el estudio Data, con 1084 pacientes en fase IV en nuestro país, mostró una excelente tolerancia y escasez de efectos secundarios durante 6 meses de seguimiento ${ }^{24}$.

\subsection{Antagonistas opiáceos}

Desde hace unos años se está investigando la relación existente entre el sistema opioide endógeno y el consumo de alcohol. Estudios de laboratorio y clínicos han sugerido la eficacia de los tratamientos con antagonistas opioides en la dependencia alcohólica. Algunos de estos estudios sostienen la disfunción endógena primaria de los sistemas de endorfinas y encefalinas en animales que espontáneamente consumen alcohol ${ }^{25,26}$ y está ampliamente aceptado que la administración de alcohol altera el contenido de $\beta$ endorfinas y encefalinas en el cerebro y glándula hipofisaria. Así mismo, las recaídas en la ingesta de alcohol podrían deberse a un nivel anormalmente bajo de $\beta$-endorfinas centrales secundarias a un fenómeno de feed-.back por el incremento agudo de $\beta$-endorfinas, producidas por el consumo crónico de alcohol ${ }^{27,28}$. Otras teorías sostienen que las propiedades reforzantes del alcohol son debidas en gran medida a la activación producida por esta sustancia en el sistema opioide endógeno y que los antagonistas opioides inhibirían la capacidad reforzante del alcohol y por lo tanto el deseo de consumir ${ }^{29}$. También se ha postulado que los inhibidores opiáceos podrían modular la actividad dopaminérgica ${ }^{10}$ que se ha relacionado estrechamente con el deseo compulsivo de beber o craving. La naltrexona reduce, por ejemplo, la liberación refleja de dopamina en el nucleus accumbens, modulando la sensibilización dopaminérgica ${ }^{30}$. Esta última hipótesis reviste gran interés ya que la sensibilización neuronal ha sido considerado como el mecanismo neurobiologico responsable de la adicción y el craving por autores como Robins y Berridge'.

\section{- Naltrexona}

La naltrexona fue el primer fármaco al que se le otorgó un efecto especifico sobre el deseo de beber o craving y su uso como tratamiento del alcoholismo se generalizó rápidamente desde su aprobación por la FDA (Food and drug administration) en 1993. A partir de los trabajos iniciales de Volpicelli ${ }^{31} \mathrm{y}$ $\mathrm{O}^{\prime}$ Malley ${ }^{32}$ se realizaron múltiples estudios con naltrexona versus placebo tanto internacionales como en nuestro país ${ }^{4,33}$. La Naltrexona reduce en todos los estudios las escalas de craving utilizadas. En el estudio de O'Malley y colaboradores ${ }^{32}$ el tipo de psicoterapia utilizado (coping skills o terapia de apoyo) condiciona la eficacia del fármaco. Los pacientes que recibieron terapia de apoyo mas naltrexona incrementaron las tasas de abstinencia total, mientras que en los pacientes que recibieron terapia cognitiva con habilidades de afrontamiento (coping skills) además de naltrexona, no se obtuvo diferencias en cuanto a abstinencia respecto al grupo control pero se disminuyó los episodios de ingesta elevada de alcohol (heavy drinking). Es interesante destacar que en el estudio de Volpicelli ${ }^{31}$ y en el de O’Malley ${ }^{32}$, la Naltrexona 
disminuye las recaídas en pacientes que prueban el alcohol, disminuyendo el número de días de consumo y las cantidades totales de alcohol. Estos estudios a pesar de tener un tiempo de seguimiento corto, mantienen un aceptable rigor metodológico y sus resultados van siendo confirmados por la mayoría de estudios mas recientes, tanto doble ciego ${ }^{33,34}$, así como por amplios estudios de farmacovigilancia ${ }^{35}$. Así mismo, un novedoso estudio realizado en nuestro medio por Rubio y colaboradores comparando un año de tratamiento con $50 \mathrm{mg} /$ dia de naltrexona versus acamprosato (1665-1998 mg/dia), demuestra incluso un mayor efecto en la disminución del número de recaídas y de los días de abstinencia acumulados con la naltrexona respecto del acamprosato ${ }^{36}$.

Por otro lado, también es importante reseñar que el trabajo de Chick y colaboradores ${ }^{37}$ no detecta diferencias en la abstinencia total entre grupos y que el más reciente estudio realizado por Krystal y colaboradores con una amplia muestra de pacientes varones ( $n=627)$ y 12 meses de tratamiento no ha podido demostrar ninguna diferencia significativa respecto el grupo placebo en el porcentaje de días con consumo y el número de bebidas alcohólicas consumidas por día de consumo ${ }^{38}$. Así mismo, el estudio de seguimiento realizado por Anton refleja una pérdida de las diferencias significativas a las 14 semanas de retirar el tratamiento ${ }^{39}$ y Kranzler publica un estudio negativo con más abandonos con naltrexona que con placebo o nefazodona por efectos secundarios de la naltrexo$n a^{40}$. El problema de la falta de cumplimiento o abandono del tratamiento por efectos secundarios o poca motivación, se ha revelado como un factor importante en la eficacia del tratamiento con naltrexona ${ }^{41,42}$ y quizás las nuevas presentaciones de este fármaco en forma de liberación retardada y subcutánea puedan, en un futuro, mejorar el cumplimiento terapéutico. Kranzler y colaboradores, en un estudio preliminar usando naltrexona depot, detectaron concentraciones en plasma de naltrexona hasta 30 días después de la inyección además de demostrar un efecto del preparado, superior a placebo, en la disminución de consumos elevados (heavy drin$k_{i n g}{ }^{43}$. Sin embargo, la eficacia de estos preparados está aún por demostrar y estudios con animales alertan de una perdida de la eficacia de la naltrexona para disminuir el consumo de alcohol, si esta se administra de forma sostenida.

Por último, la posibilidad de la Naltrexona de reducir la intensidad del consumo de alcohol en pacientes alcohólicos no abstinentes, abre interesantes posibilidades terapéuticas en el ámbito de reducción de daños que están aún por explorar.

\section{- Nalmefene}

El Nalmefene es un antagonista opiáceo específico para el receptor delta que se presenta como una posible alternativa a la Naltrexona. Existen dos estudios con este fármaco, el primero, con una muestra pequeña y dos dosis de nalmefene (10 y 40 mg/día) aunque no aumentó la abstinencia respecto a placebo, sí disminuyó los episodios de consumo severo de alcohol. Posteriormente, los mismos autores estudiaron una muestra más amplia con dosis de $20 \mathrm{mg} /$ día y $80 \mathrm{mg} /$ día obteniendo resultados parecidos ${ }^{44}$. Con todo, los estudios con este fármaco son aún muy preliminares y su utilidad y seguridad clínica siguen pendientes de confirmación.

\subsection{Fármacos dopaminérgicos}

La importancia de la transmisión dopaminérgica en las vías responsables del refuerzo positivo en animales está ampliamente aceptada. En prácticamente todas las drogas de abuso se ha demostraron un incremento de la liberación dopaminérgica en el nucleus accumbens ${ }^{44}$. Se conoce también la estrecha relación funcional existente entre el sistema opioide y el dopaminérgico, como ya se ha comentado previamente. En modelos animales, el consumo crónico de alcohol produce una alteración en la neurotransmisión dopaminérgica, una disminución de la liberación de dopamina y probablemente una sensibili- 
zación de los receptores que podrían ser según algunos autores las bases neurobiológicas de la adicción'.

\section{- Bromocriptina}

Se trata de un agonista dopaminérgico de acción prolongada. A pesar de que los estudios con animales demuestran una reducción del consumo de alcohol y los primeros estudios clínicos parecían prometedores, la publicación por Naranjo y colaboradores ${ }^{45}$ de un estudio multicéntrico con una muestra mas amplia de pacientes y un mayor seguimiento, pone en duda su eficacia. El estudio de Lawford con 83 pacientes propone un efecto selectivo de la bromocriptina para los portadores del alelo A1 del gen para el receptor D2 dopaminérgico ${ }^{46}$.

\section{- Antagonistas dopaminérgicos}

Tiapride es un antagonista débil de los receptores D2 que carece de los típicos efectos secundarios de los neurolépticos. Algunos estudios han demostrado un cierto efecto en pacientes alcohólicos pero las muestras son pequeñas y por el momento se requieren aún estudios más amplios ${ }^{47}$. Otros antipsicóticos, como el flupentixol han sido también estudiados, y aunque inicialmente los resultados preliminares parecían esperanzadores ${ }^{48}$ un reciente estudio con una muestra de 281 pacientes y un seguimiento de 6 meses con $10 \mathrm{mg}$ de flupentixol no pudo demostrar ninguna eficacia de este fármaco para mantener la abstinencia al alcohol ${ }^{49}$.

\subsection{Fármacos serotoninérgicos}

Un importante número de estudios, apuntan hacia una disfunción en la neurotransmisión serotoninérgica en al menos un subgrupo de alcohólicos. Se ha descrito una disminución del 5 HIAA, un metabolito de la serotonina, en el líquido cefalorraquídeo de pacientes alcohólicos y se postula que al incrementar el alcohol la liberación serotoninérgica, su consumo podría normalizar los niveles de serotonina en el SNC. La disminu- ción de serotonina también está implicada en fenómenos como la impulsividad, la ansiedad o la depresión, trastornos que podrían predisponer a los pacientes al consumo y a la recaída alcohólica.

\section{- Inhibidores selectivos de la recaptación de la serotonina (ISRS)}

Son muchos los estudios que se han llevado a cabo con estos fármacos, pero en general los resultados obtenidos han sido poco satisfactorios ${ }^{20}$. Parece que el efecto beneficioso de los ISRS estaría en un subgrupo determinado de pacientes. Naranjo y colaboradores ${ }^{50}$ describen una reducción del craving aunque no una diferencia en las tasas de abstinencia en el tratamiento con fluoxetina en los pacientes con alcoholismo moderado. Otro subgupo específico de pacientes serían los alcohólicos con síndrome depresivo que mejorarían no sólo de su sintomatología depresiva, sino también las tasas de recaída y consumo de alcohol ${ }^{51}$. Por otro lado, los estudios más recientes son aún contradictorios y por ejemplo tenemos un estudio realizado por Kranzler en 1995 con una muestra de 101 alcohólicos tratados con fluoxetina que no encuentra diferencias contra placebo y un estudio realizado por Cornelius en 1997 con 51 pacientes que sí detecta diferencias significativas en el tratamiento con fluoxetina contra placebo ${ }^{52,53}$.

Así mismo también se están utilizando otros IRSS como el citalopram y la sertrali$\mathrm{na}^{42,54,55}$. Estudios en pacientes alcohólicos deprimidos con otros ISRS como la Sertralina se han finalizado recientemente en nuestro medio, pero los resultados están aún por determinar ${ }^{56}$.

\section{- Buspirona}

La buspirona es un agonista 5-HT1A con perfil ansiolítico. Los estudios con este fármaco realizados en pacientes alcohólicos que presentan trastornos concomitantes de ansiedad no confirman su posible eficacia ${ }^{57}$. Malec y colaboradores realizaron un meta-análisis con los cinco estudios publicados hasta 1996 poniendo en evidencia que la buspirona, en el 
tratamiento del alcoholismo, tenía un efecto solo en las tasas de retención al tratamiento. Así mismo, este efecto de la buspirona parecía mas asociado a la reducción de sintomatología psicopatológica que a la modificación del craving ${ }^{58}$. Por último, Linnoila y su grupo, en un reciente estudio doble ciego, en el que los pacientes recibieron buspirona durante todo un año, no pudieron demostrar ningún efecto significativo para la buspirona ni en el tiempo hasta la primera recaída ni en la adhesión al tratamiento ${ }^{59}$.

\section{- Ondansetron}

El ondansetron es un fármaco serotoninérgico que actúa a nivel del receptor 5-HT3 y que se usa como potente antiemético. En algunas hipótesis, el receptor serotoninérgico 5-HT3 se ha relacionado con las conductas adictivas. Johnson y colaboradores han realizado un reciente estudio en alcohólicos de inicio precoz y con carga genética familiar de alcoholismo con ondansetron más naltrexona y terapia grupal, en el que detectan una disminución de la cantidad y frecuencia de consumo de alcohol con esta combinación terapéutica ${ }^{60,61}$.

\section{ASOCIACIONES FARMACOLÓGICAS}

La diversidad de fármacos existentes con una disparidad en su mecanismo de acción, junto con la eficacia tan solo parcial o reducida de todos ellos ha llevado a algunos autores a investigar la posibilidad y la eficacia de combinar distintos fármacos en un mismo tratamiento. La combinación de distintos fármacos para tratar una enfermedad tan compleja y multicausal como el alcoholismo puede tener sin duda cierta base científica pero deberemos ser también sobradamente cautos al empezar a promover esta práctica clínica tan de moda en otras ramas de la psiquiatría. Los estudios clínicos para evaluar la eficacia de combinaciones terapéuticas deben estar correctamente diseñados y poseer suficiente potencia estadística para poder suministrar conclusiones válidas. Por el momento, los estudios de investigación básica con animales no han demostrado ninguna sinergia al combinar distintos fármacos ${ }^{62}$. Los resultados del estudio COMBINE que compara la utilización en un estudio clínico naltrexona y acamprosato solos o en combinación están aun por publicar y no disponemos por el momento de datos preliminares sobre las ventajas terapéuticas de esta combinación.

\section{CONCLUSIÓN}

La dependencia al alcohol es una enfermedad progresiva, crónica y altamente recidivante que por su elevada prevalencia y su alta morbi-mortalidad se ha convertido en uno de los principales problemas de salud pública en nuestro medio.

En el tratamiento farmacológico del alcoholismo los compuestos aversivos siguen estando vigentes y se consideran eficaces sobretodo si van acompañados de supervisión y de otros tratamientos de soporte y psicológicos. Dos nuevos fármacos, la Naltrexona y el Acamprosato, avalados por investigaciones básicas y estudios clínicos controlados y rigurosos, están ya disponibles en la clínica diaria. Estos fármacos, actuando por vías y mecanismos distintos, disminuirían el número de recaídas y reducirían el craving o deseo irrefrenable de beber. Otros compuestos se perfilan como importantes en el tratamiento del alcoholismo en pacientes con sintomatología psiquiátrica concomitante (depresión, ansiedad).

En el futuro deberá identificarse mejor los subgrupos de enfermos que pueden beneficiarse de cada uno de los fármacos psicotropos disponibles y realizar combinaciones terapéuticas para abordar cada una de las alteraciones subyacentes. No hay que olvidar pero, que un tratamiento multidisciplinario con profesionales con un alto nivel de capacidad de empatía que sepan explicar motivar y aconsejar, seguirá siendo la base de la terapia del alcoholismo. 


\section{REFERENCIAS BIBLIOGRÁFICAS}

(1) Robinson TE, Berridge KC. The neural basis of drug craving: an incentive-sensitization theory of addiction. Brain Res Review 1993; 18:247291.

(2) Hughes JC, Cook CH. The efficacy of disulfiram; a review of outcome studies. Addiction 1997, 92 (4): 381-395.

(3) Azrin NH, Sisson RW, Meyers R, Godley M. Alcoholism treatment by disulfiram and community reinforcement therapy. J Behav Ther Exp Psych 1982, 13:105-112.

(4) Landabaso MA, Iraurgi I, Sanz J, Fernandez de Corres B, Ruiz de Apodaka J, Jiménez-Lerma JM, Calle R, Araluce K, Gutierrrez-Fraile M. Naltrexona mas aversivo en alcoholicos refractarios a tratamiento. Psiquiatría Biológica 1997; 4:5-8.

(5) Chick J, Gough K, Falkowski et al. Disulfiram treatment of alcoholism. Br J Psy 1992; 161:84-9.

(6) Wilson A, Davidson WJ, Blanchart R, Withe J. Disulfiram implantation: a placebo-controlled trial with two-year follow-up. J Studies Alcohol 1978; 39:809-819.

(7) Wilson A, Davidson WJ, Blanchart R, Withe J. Disulfiram implantation: a trial using placebo implants and two types of controls. J Studies Alcohol 1980; 41:429-436.

(8) Fuller R, Branchey L, Brightwell D, Derman R, Emrick $C$, Iber F, James $K$, Lacoursiere R, Lee K, Lowenstam I, Maany I, Neiderhiser D, Nocks J, Shaw S. Disulfiram treatment of alcoholism: A Veterans Administration cooperative study. Journal American Medical Association 1986. 256:1449-1455.

(9) Soyka M. Pharmacological trials in alcoholics: some comments on methodological problems. Addiction 1997; 92:956-957.

(10) Samson HH, Harrris RA. The neurobiology of alcohol abuse. TINS 1992; 13:206-211.

(11) Addolorato G, Fibin M, Caprista E, Beghe F, Gessa G, Stefanini GF, Gasbarrini G. Maintaining abstinence from alcohol with $\gamma$ hidroxibutyric acid. Lancet 1998; 351:38.

(12) Gallimberti L, Ferri M, Ferrara SD, Fada S, Gessa GL. g-hidroxibutyric acid in the treatment of alcohol dependence: a double blind study. Alcohol Clin Exp Res 1992; 16:673-676.
(13) Addolorato G, Caputo F, Stefanini GF, Gasbarrini G. g-hidroxibutyric acid in the treatment of alcohol dependence: possible craving development for the drug. Addiction 1997; 92:10411042.

(14) Hunderup MG and Jorgensen AJ. Poisoning with gamma-hydroxybutyrate. Cases reported in connection with "cultural festivals" in August 1999 in Kolding]. Ugeskr Laeger. 1999 Dec 13; 161(50):6939-40. Danish.

(15) Bowles TM, Sommi RV, Amiri M. Successful management of prolonged gamma-hydroxybutyrate and alcohol withdrawal. Pharmacotherapy. 2001 Feb;21(2):254-7.

(16) Moncini M, Masini E, Gambassi F, Mannaioni PF. Gamma-hydroxybutyric acid and alcoholrelated syndromes. Alcohol. 2000 Apr;20(3): 285-91.

(17) Littleton J. Acamprosate in alcohol dependence; how does it work? Addiction 1995; 90:1179-88.

(18) Hilemand B, Lhuintre JP, Boismare F, Daoust M, Moore N. Premier essai favorable du bis acetylhomotaurinate de calcium (AOTA Ca) dans le traitement de l'alcoolodependant. Rev Alcool 1988; 30:204-212.

(19) Moncrieff J, Drummond DC. New drug treatment for alcohol problems: a critical appraisal. Addiction 1997; 92:939-947.

(20) Soyka M. Relapse prevention in alcoholism. Recent advances and future possibilities. Drug Therapy 1997; 7:313-327.

(21) Withworth $A$ et al. Acamprosate versus placebo in the long term treatment of patients with dependence. Lancet 1996; 347:1438-1442.

(22) Aubin HJ. Acamprosate in clinical practice: the french experience. In: Acamprosate in relapse prevention of alcoholism. Soyka (ed). Springer. Berlin 1996, pp 111-120.

(23) Gual A [Naltrexone in the treatment of alcoholism. Clinical evolution, safety and efficacy in a sample of 198 patients]. Med Clin (Barc). 2001 Apr 21;116(14):526-32.

(24) Gual A and Balcells-Olivero M Estudio DATA. Data on File. Merck S.A.

(25) De Waele JP, Papachristou DN, Gianoulakis C. The alcohol prefering C57BI/6 mice present an enhanced sensitivity of the hypothalamic bendorphin system to ethanol than the alcohol avoiding DBA/2 mice. J Pharmacol Exp Ther 1992; 261:788-794. 
(26) Gianoulakis C, De Waele JP, Kianmaa K. Differences in the brain and pituitary b-endorphin system between the alcohol preferent AA and alcohol avoiding ANA rats. Alcohol Clin Exp Res 1992; 16:453-459.

(27) Genazzani AR, Nappi G, Facchinetti F et al. Central deficiency of b-endorfine in alcohol addicts. J Clin Endocrinol Metab 1982; 55,3: 583-586.

(28) Gianoulakis C, De Waele JP, Thavundayil J. Implication of the endogenous opioid system in excessive ethanol consumption. Alcohol 1996; 13, 1:19-23.

(29) Anton RF, Kranzler HR, Meyer RE. Neurobehavioral aspects of pharmacotherapy of alcohol dependence. Clin Neuroscience 1995; 3:145154.

(30) Balcells-Olivero M, Vezina P. Effects of naltrexone on amphetamine-induced locomotion and rearing: acute and repeated injection. Psychopharmacology 1997; 131:230-238.

(31) Volpicelli JR, Alterman Al, Hayashida M et al. Naltrexone in the treatment of alcohol dependence. Arch Gen Psychiatry 1992; 49:876-880.

(32) O'Malley SS, Jaffe AJ, Chang G, Scottenfeld RS, Meyer RE, Rounsaville B. Naltrexone and coping skills therapy for alcohol dependence. Arch Gen Psychiatry 1992; 49:881-887.

(33) Guardia J, et al. A double-blind, placebo-controlled study of naltrexone in the treatment of alcohol-dependence disorder. Results from a multicenter clinical trial. Americal Journal. Submited

(34) Anton RF, Moak DH, Waid LR, Latham PK, Malcolm RJ, Dias JK. Naltrexone and cognitive behavioral therapy for the treatment of outpatient alcoholics: results of a placebo-controlled trial. Am J Psychiatry. 1999 Nov;156(11):175864.

(35) Gual A, Lehert P.Acamprosate during and after acute alcohol withdrawal: a double-blind placebo-controlled study in Spain. Alcohol 2001 SepOct;36(5):413-8.

(36) Rubio G, Jimenez-Arriero MA, Ponce G, Palomo T. Naltrexone versus acamprosate: one year follow-up of alcohol dependence treatment. Alcohol. 2001 Sep-Oct;36(5):419-25.

(37) Chick J. UK multicentre study of naltrexone as adjuntive therapy in the treatment of alcoholism: efficacy results. Abstracts of the Xth world congress of psychiatry 1996; 1:230.
(38) Krystal JH, Cramer JA, Krol WF, Kirk GF, Rosenheck RA. Naltrexonein the treatment of alcohol dependence. New Engl J Med 2001; 345:173439.

(39) Anton RF, Moak DH, Latham PK, Waid LR, Malcolm RJ, Dias JK, Roberts JS. Posttreatment results of combining naltrexone with cognitivebehavior therapy for the treatment of alcoholism. J Clin Psychopharmacol 2001 Feb;21(1): 72-7.

(40) Kranzler HR, Modesto-Lowe V, Van Kirk J. Naltrexone vs. nefazodone for treatment of alcohol dependence. A placebo-controlled trial. Neuropsychopharmacology. 2000 May;22(5): 493-503.

(41) Chick J, Anton R, Checinski K, Croop R, Drummond DC, Farmer R, Labriola D, Marshall J, Moncrieff J, Morgan MY, Peters T, Ritson B. A multicentre, randomized, double-blind, placebo-controlled trial of naltrexone in the treatment of alcohol dependence or abuse. Alcohol Alcohol. 2000 Nov-Dec;35(6):587-93.

(42) Pettinati HM; Volpicelli JR; Luck G; Kranzler HR; Rukstalis MR; Cnaan A. Double-blind clinical trial of sertraline treatment for alcohol dependence. J Clin Psychopharmacol (United States), Apr 2001, 21 (2) p143-53.

(43) Kranzler HR, Modesto-Lowe V, Nuwayser ES. Sustained-release naltrexone for alcoholism treatment: a preliminary study. Alcohol Clin Exp Res. 1998 Aug;22(5):1074-9.

(44) Mason B. Nalmefene modification of alcohol dependence. Joint scientific meeting 8th ISBRA congress and RSA meeting. International update: New findings on promising medications. 1996.

(45) Naranjo CA, Dongier M, Bremner KE. Longacting injectable bromocriptine do not reduce relapse in alcoholics. Addiction 1997; 92:969978.

(46) Lawford BR, Young RM, Rowell JA et al. Bromocriptine in the treatment of alcoholic with the $D_{2}$ dopamine receptor allele. Nature Med 1995; 1:337-341.

(47) Shaw GK, Waller SK, Majumdar JL, Latham CJ, Dunn G. Tiapride in the prevention of relapse in recently detoxified alcoholics. Br J Psych 1994; 165: 515-523.

(48) Balldin J, Berggren U, Bokstrom K, et al. Sixmonth open trial with zimelidine in alcoholdependent patients: reduction in days of alcohol intake. Drug Alcohol Depend 1994; 35:245-248. 
(49) Wiesbeck GA, Weijers HG, Lesch OM, Glaser T, Toennes PJ, Boening J Flupenthixol decanoate and relapse prevention in alcoholics: results from a placebo-controlled study. Alcohol Alcohol. 2001 Jul-Aug;36(4):329-34.

(50) Naranjo CA, Kadlec KE, Sanhueza P et al. Fluoxetine differentially alters alcohol intake and other consummatory behaviors in problem drinkers. Clin Pharmacol Ther 1990; 47:490-498.

(51) Cornelius JR, Salloum IM, Cornelius MD et al. Fluoxetine trial in suicidal depressed alcoholics. Psychopharmacol Bull 1993; 29:195-199.

(52) Cornelius JR, Salloum IM, Ehler JG, Jarrett PJ, Cornelius MD, Perel JM, Thase ME, Black A. Fluoxetine in depressed alcoholics. A doubleblind, placebo-controlled trial Arch Gen Psychiatry. 1997 Aug; 54(8):700-5.

(53) Kranzler HR, Burleson JA, Brown J, Babor TF. Fluoxetine treatment seems to reduce the beneficial effects of cognitive-behavioral therapy in type $\mathrm{B}$ alcoholics. Alcohol Clin Exp Res, Dec 1996, 20(9) p1534-41.

(54) Tiihonen J, Ryynanen OP, Kauhanen J, Hakola HP, Salaspuro M. Citalopram in the treatment of alcoholism: a double-blind placebo-controlled study. Pharmacopsychiatry 1996 Jan;29(1):27-9.

(55) Roy A. Placebo-controlled study of sertraline in depressed recently abstinent alcoholics. Biol Psychiatry (United States), Oct 1 1998, 44(7) p633-7.
(56) Gual A, Balcells M. Eficacia de sertralina frente a placebo en el tratamiento de alcohólicos deprimidos. Data on file. Pfizer S.A.

(57) Kranzler HB, Burleson JA, Del Boca FK et al. Buspirone treatment of anxious alcoholics: a placebo-controlled trial. Arch Gen Psychiatry 1994; 51:720-731.

(58) Malec TS, Malec EA, Dongier M. Efficacy of buspirone in alcohol dependence: a review. Alcohol Clin Exp Res, Aug 1996, 20(5) p853-8.

(59) George DT, Rawlings R, Eckardt MJ, Phillips MJ, Shoaf SE, Linnoila M. Buspirone treatment of alcoholism: age of onset, and cerebrospinal fluid 5-hydroxyindolacetic acid and homovanillic acid concentrations, but not medication treatment, predict return to drinking. Alcohol Clin Exp Res, Feb 1999, 23(2) p272-8.

(60) Johnson BA, Ait-Daoud N, Prihoda TJ. Combining ondansetron and naltrexone effectively treats biologically predisposed alcoholics: from hypotheses to preliminary clinical evidence. Alcohol Clin Exp Res. 2000 May;24(5):737-42.

(61) Ait-Daoud N, Johnson BA, Prihoda TJ, Hargita ID. Combining ondansetron and naltrexone reduces craving among biologically predisposed alcoholics: preliminary clinical evidence. Psychopharm (Berl) 2001; 154(1):23-7.

(62) Stromberg MF, Mackler SA, Volpicelli JR, O'Brien CP. Effect of acamprosate and naltrexone, alone or in combination, on ethanol consumption. Alcohol, Feb 2001, 23(2) p109-16. 